\title{
Controlling rotation in the molecular frame with an optical centrifuge
}

\author{
Emil J. Zak $\odot,{ }^{1, *}$ Andrey Yachmenev $\odot,{ }^{1,2}$ and Jochen Küpper $\circledast^{1,2,3, \dagger}$ \\ ${ }^{1}$ Center for Free-Electron Laser Science, Deutsches Elektronen-Synchrotron DESY, Notkestraße 85, 22607 Hamburg, Germany \\ ${ }^{2}$ Center for Ultrafast Imaging, Universität Hamburg, Luruper Chaussee 149, 22761 Hamburg, Germany \\ ${ }^{3}$ Department of Physics, Universität Hamburg, Luruper Chaussee 149, 22761 Hamburg, Germany
}

(Received 8 September 2020; revised 28 April 2021; accepted 4 May 2021; published 7 June 2021)

\begin{abstract}
We computationally demonstrate a new method for coherently controlling the rotation-axis direction in asymmetric top molecules with an optical centrifuge. Appropriately chosen electric-field strengths and the centrifuge's acceleration rate allow to generate a nearly arbitrary rotational wave packet. For dihydrogen sulfide $\left(\mathrm{D}_{2} \mathrm{~S}\right)$ and $2 \mathrm{H}$-imidazole $\left(\mathrm{C}_{3} \mathrm{H}_{4} \mathrm{~N}_{2}\right)$, we created wave packets at large values of the rotational quantum number $J$ with the desired projections of the total angular momentum onto two of the molecules' principal axes of inertia. One application of the new method is three-dimensional alignment with a molecular axis aligned along the laser's wave vector, which is important for the three-dimensional imaging of molecules yet not accessible in standard approaches. The simultaneous orientation of the angular momentum in the laboratory frame and in the molecular frame could also be used in robust control of scattering experiments.
\end{abstract}

DOI: 10.1103/PhysRevResearch.3.023188

\section{INTRODUCTION}

Preparing well-defined molecular samples, maximally fixed in three-dimensional space, has been a long-term goal in physics, chemistry, and related fields such as quantum technology [1-4]. Significant progress toward this goal was made in the gas phase using internally cold molecular samples and tailored external fields [5-8]. Nowadays, it is possible to strongly align and orient molecules in the laboratory frame in one [9-12], two [13-15], and three [16-20] spatial directions. It is also possible to create unidirectionally rotating molecules with oriented-in-space angular momentum and a narrow rotational energy spread [21-24]. Such alignment and orientation of molecules is critical for imaging molecular structure and ultrafast dynamics in the molecule-fixed frame [25-32] as well as in the stereodynamical control of chemical reactions [33-36].

A highly efficient technique used to generate and control the molecule's angular momentum is the optical centrifuge $[37,38]$, which is a strong nonresonant linearly polarized laser pulse that performs accelerated rotation of its polarization about the direction of propagation. It can excite molecules into rotational states with extremely large angular momentum, creating an ensemble of superrotors [39,40]. Molecules in superrotor states are aligned in the polarization plane of

\footnotetext{
*emil.zak@cfel.de

†jochen.kuepper@cfel.de; website: https://www.controlled-
} molecule-imaging.org

Published by the American Physical Society under the terms of the Creative Commons Attribution 4.0 International license. Further distribution of this work must maintain attribution to the author(s) and the published article's title, journal citation, and DOI. the centrifuge [23] and resist collisional decoherence for microseconds [39,41-43].

The optical centrifuge can also be utilized as a versatile tool to fine tune the rotational dynamics of molecules, including the coherent control of the rotation axis [44], the enantiomerspecific excitations of chiral molecules [45-47], and even the creation of chiral samples from achiral molecules [24].

Here, we further explore the possibility of tailoring the optical field to steer the rotational dynamics of asymmetric top molecules. We computationally demonstrate a new type of rotational coherent control by exciting the rotation of an asymmetric top molecule about two different axes of inertia with simultaneously fully controlled orientation of the angular momentum in both, the laboratory-fixed and the molecule-fixed, frames.

We apply our method to asymmetric top molecule $\mathrm{D}_{2} \mathrm{~S}$, which exhibits the effects of rotational energy level clustering and dynamical chirality at high rotational excitations $[24,44,48]$. To populate the rotational cluster states, $D_{2} S$ has to be excited along a specific pathway of rotational states. Previously, we explored the method of pulse shaping, i.e., by repeatedly turning on and off the field, to make the asymmetric top molecule $\mathrm{H}_{2} \mathrm{~S}$ rotate about either of its two stable inertial axes. We demonstrated that only the $a$-axis rotational excitation leads to the population of rotational cluster states in $\mathrm{H}_{2} \mathrm{~S}$. Here, we propose a more robust approach to create arbitrary coherences between the two stable molecular rotations, which relies only on the careful selection of the centrifuge angular acceleration rate. The heavier $\mathrm{D}_{2} \mathrm{~S}$ isotopologue is chosen for the present study due to the lower laser intensities required for efficient rotational excitation, thus reducing potential ionization. Along with robust quantummechanical calculations, we derived a simple analytical metric that allows to predict the orientation of the angular momentum 

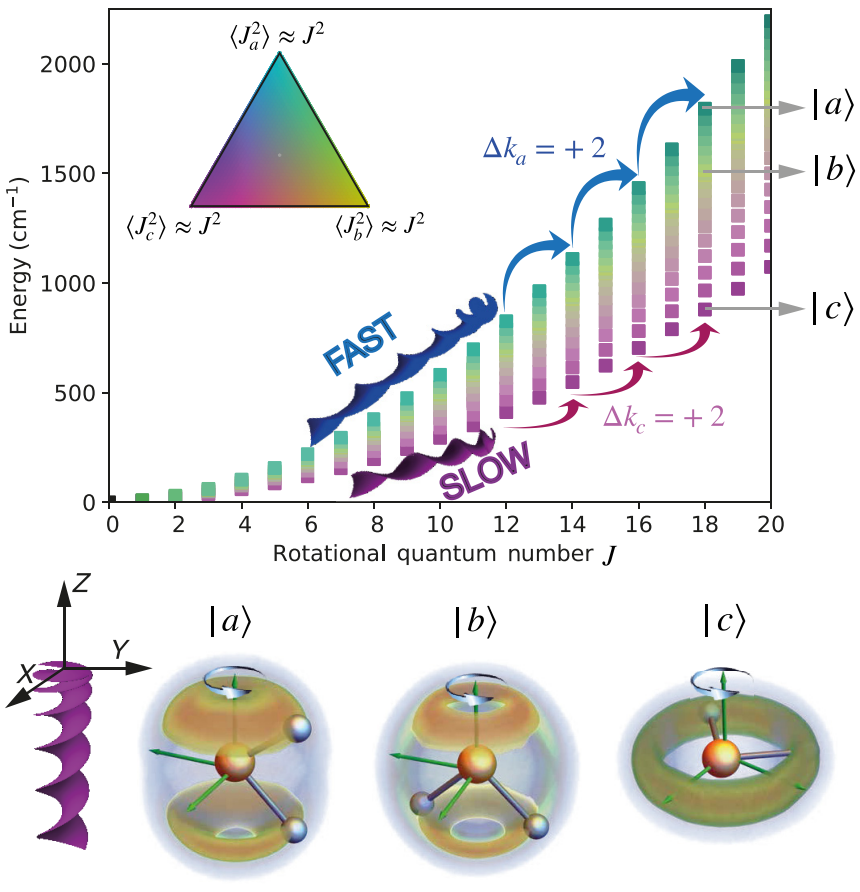

FIG. 1. (Top) Rotational-energy-levels of the near-oblate asymmetric top molecule $\mathrm{D}_{2} \mathrm{~S}$ as a function of the rotational quantum number $J$. Each level is color-coded depending on its expectation values $\left\langle\hat{J}_{a}^{2}\right\rangle,\left\langle\hat{J}_{b}^{2}\right\rangle$, and $\left\langle\hat{J}_{c}^{2}\right\rangle$ according to the color map shown in the inset. Arrows schematically mark the two competing excitation pathways "fast" and "slow." Three principal rotation states for $J=$ 18 and $M=18$ are denoted as $|a\rangle,|b\rangle$, and $|c\rangle$; see text for details. (Bottom) 3D probability-density distributions of the deuterium atoms for three selected principal rotation states. In the laboratory frame, the centrifuge pulses propagate along the laboratory $Z$ axis, trap two of the molecule-fixed axes in the $X Y$ plane and unidirectionally spin the molecules about the third axis, which is aligned along $Z$.

in the molecule-fixed frame for arbitrary molecules given the parameters of the optical centrifuge.

Additionally, by adjusting the turn-off time we demonstrate the two types of three-dimensional (3D) alignment of asymmetric top molecules, with either of the two stable rotation axes pointing along the field's wave vector, so called $k$ alignment $[13,14,49]$.

\section{PRINCIPAL-ROTATION STATES}

Figure 1 displays the rotational energy level structure of the $\mathrm{D}_{2} \mathrm{~S}$ molecule. For any value of the rotational quantum number $J$ there is a multiplet of $2 J+1$ levels, which are colored according to the average values of the angular momentum projection operators onto the principal axes of inertia $\left\langle\hat{J}_{a}^{2}\right\rangle,\left\langle\hat{J}_{b}^{2}\right\rangle$, and $\left\langle\hat{J}_{c}^{2}\right\rangle$. The bottom panel in Fig. 1 shows the calculated $3 \mathrm{D}$ probability density for deuterium atoms for the highest-, middle-, and lowest-energy levels at $J=$ 18 and $M=18$. Here, $M$ is the quantum number for the $Z$ component of the angular momentum operator in the laboratory-fixed frame. It is evident that the highest-energy levels within each $J$ multiplet correspond to $\left\langle\hat{J}_{a}^{2}\right\rangle \approx J^{2}$ (cyan color), i.e., in these states the molecule rotates about the $a$ axis and $k_{a}=J$ becomes a near-good quantum number. The lowest-energy levels correspond to rotation about the $c$-axis with $k_{c}=J$ (purple color), while those with energies in the middle are mixtures of rotations about different axes with some of them exhibiting classically unstable $b$-axis rotation (yellow color).

We refer to principal-rotation states when the rotational angular momentum is nearly aligned along one of the principal axis of inertia, i.e., $k_{a}=J, k_{c}=J$, or $k_{b}=J$. Controlling populations of the principal rotation states, allows to create arbitrary three-dimensional orientation of the total angular momentum in the molecule-fixed frame. We investigated the orientation of the angular momentum in the $a c$ plane by controlling the populations of the lowest- and highest-energystate components in the rotational wave packet.

Principal rotation states can be populated through the interaction with the optical centrifuge field, represented by

$$
\mathbf{E}(t)=E_{0} f(t) \cos (\omega t)\left[\mathbf{e}_{X} \cos \left(\beta t^{2}\right)+\mathbf{e}_{Y} \sin \left(\beta t^{2}\right)\right],
$$

with the peak amplitude $E_{0}$, the pulse envelope $f(t)$, the acceleration $\beta$ of angular rotation of the polarization, and the far off-resonant ( $\lambda=800 \mathrm{~nm}$ ) carrier frequency $\omega$ of the linearly polarized pulse. A molecule placed in the optical centrifuge field experiences a series of Raman transitions with $\Delta J=2$ and $\Delta m= \pm 2$, depending on the sign of $\beta$, which defines the direction of the centrifuge rotation.

Initially in the rotational ground state [8], the molecule can undergo two main excitation pathways: along the lowestenergy or along the highest-energy rotational states of the $J$ multiplets. Excitation rates for these two pathways are governed by the two specific polarizability-interaction terms in the molecule-field interaction potential:

$$
\begin{aligned}
V(t)= & \frac{1}{4} E_{0}^{2} f(t)^{2} \cos ^{2}(\omega t) \\
& \times\left[-\Delta \alpha_{1}\left(D_{2,0}^{(2) *} e^{-2 i \beta t^{2}}+D_{-2,0}^{(2) *} e^{2 i \beta t^{2}}-\sqrt{\frac{2}{3}} D_{0,0}^{(2) *}\right)\right. \\
& \left.-\Delta \alpha_{2}\left(S_{2,2}^{(2) *} e^{-2 i \beta t^{2}}+S_{-2,2}^{(2) *} e^{2 i \beta t^{2}}-\sqrt{\frac{2}{3}} S_{0,2}^{(2) *}\right)\right] .
\end{aligned}
$$

Here, $D_{M, k}^{(J) *}$ denotes the complex-conjugated Wigner $D$ matrix and $S_{M, k}^{(2) *}=D_{M, k}^{(2) *}+D_{M,-k}^{(2) *}$. The expressions for $\Delta \alpha_{1}$ and $\Delta \alpha_{2}$ depend on the choice of the quantization axis in the moleculefixed frame.

A molecule is called (near) prolate if the quantization axis is along the $a$ axis and (near) oblate if it is along the $c$ axis. The first term in (2) describes the interaction with the polarizability anisotropy, $\Delta \alpha_{1}=\frac{1}{\sqrt{6}}\left(2 \alpha_{a a}-\alpha_{b b}-\alpha_{c c}\right)$ for (near-)prolate and $\Delta \alpha_{1}=\frac{1}{\sqrt{6}}\left(2 \alpha_{c c}-\alpha_{a a}-\alpha_{b b}\right)$ for (near-)oblate top. This term yields $\Delta k_{a}=0$ transitions along the lowest-energy pathway of rotational states for (near-)prolate top molecules and $\Delta k_{c}=0$ transitions along the highest-energy pathway for (near-)oblate top molecules. The second term in (2), with $\Delta \alpha_{2}=\frac{1}{2}\left(\alpha_{b b}-\alpha_{c c}\right)$ for (near-)prolate and $\Delta \alpha_{2}=\frac{1}{2}\left(\alpha_{a a}-\right.$ $\alpha_{b b}$ ) for (near-)oblate top, gives $\left|\Delta k_{a}\right|=2$ and $\left|\Delta k_{c}\right|=2$ transitions corresponding to the highest-energy and lowest-energy pathways for (near-)prolate and (near-)oblate top molecules, respectively. 
near-prolate

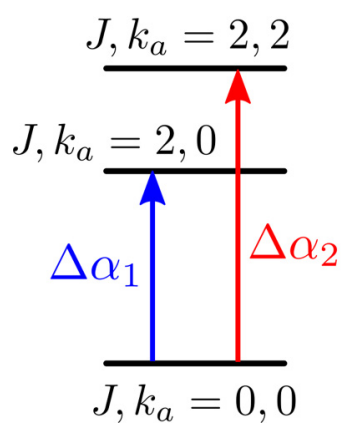

near-oblate

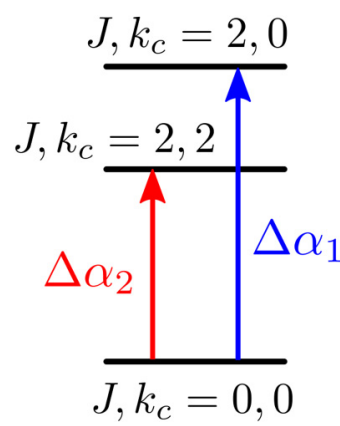

FIG. 2. Scheme of the centrifuge driven transitions from $J=$ 0 to $J=2$ states in near-prolate and near-oblate top molecules. The symbols $\Delta \alpha_{1}$ and $\Delta \alpha_{2}$ represent the transition moments defined in (2).

The ability to control the excitation pathway and ultimately the orientation of the angular momentum in the molecule-fixed $a c$ plane depends on the relationship between the molecular polarizability anisotropies $\Delta \alpha_{1}$ and $\Delta \alpha_{2}$, see Fig. 2. For a great majority of molecules it applies that $\left|\Delta \alpha_{1}\right|>\left|\Delta \alpha_{2}\right|$, and we assume this in the discussion below. Hence, for a near-prolate top molecule, the transition moment into the lowest-energy state is greater than that into the highest-energy state and vice versa for a near-oblate top molecule.

A molecule in the optical centrifuge climbs the rotational energy level ladder via a series of consecutive $\Delta J=2$ excitations between neighboring rotational states with $J=$ $0,2,4, \ldots$ Starting from $J=0$, the resonance of the centrifuge's frequency $\omega(t)=\beta t$ with the lowest-energy state in the $J=2$ multiplet occurs earlier in time than the resonance with the highest-energy state in the same multiplet. Thus, in order to steer the excitation along the highest-energy pathway in the near-prolate case one would suppress the stronger lower-frequency transitions by applying shaped pulses, i.e., by repeatedly decreasing and increasing the field intensity $f(t)$ at the crossing times with unwanted and desired transitions, respectively [44]. In the near-oblate case the highest-energy excitation pathway is favorable. However, it is possible to guide it along the lowest-energy path by only adjusting the centrifuge peak field strength $E_{0}$ and acceleration rate $\beta$. In light of recent experiments [50], such an approach seems more feasible than the pulse-intensity shaping strategy.

We investigate the possibility of controlling the rotational wave-packet composition in near-oblate top molecules by selectively populating the principal rotation states using the optical centrifuge with appropriately chosen intensity and acceleration rate $\beta$. For small $\beta$ values and high intensities the centrifuge's rotating field will first slowly cross through resonance with the ground- to lowest-excited rotational-energy level transition. If the corresponding transition moment $\sim\left|\Delta \alpha_{2}\right|^{2}$ is not entirely negligible, it will predominantly populate the $c$-axis principal rotation states. On the other hand, for large $\beta$ values and low intensities the centrifuge will chirp through resonance with low-energy $c$-axis principal rotation states fast enough to not populate them significantly. As a result the stronger transition to the $a$-axis principal rotation states will dominate, see Fig. 2 and Appendix A for a more detailed discussion. By choosing intermediate values for $\beta$, an arbitrary coherent rotational wave packet over $a$ - and $c$-axis principal rotation states can be tailored.

\section{COMPUTATIONAL DETAILS}

We computationally demonstrate the proposed technique for the near-oblate asymmetric top molecules $\mathrm{D}_{2} \mathrm{~S}$ and $2 \mathrm{H}$-imidazole $\left(\mathrm{C}_{3} \mathrm{H}_{4} \mathrm{~N}_{2}\right)$. The latter has been chosen to examine the effectiveness of our technique for larger molecules, i.e., with rotational constants smaller than in $\mathrm{D}_{2} \mathrm{~S}$ and larger electronic polarizabilities. Our calculations employed a highly accurate variational approach. The rotational-dynamics calculations of $\mathrm{D}_{2} \mathrm{~S}$ and $2 \mathrm{H}$-imidazole were performed in two steps. In the first step, the molecular field-free energies and their transition moments were obtained. For $\mathrm{D}_{2} \mathrm{~S}$ we utilized the full-dimensional variational procedure TROVE [51-53] together with a highly-accurate spectroscopically adjusted potential energy surface [54] and high-level $a b$ initio polarizability surface [44] of the $\mathrm{H}_{2} \mathrm{~S}$ molecule within the Born-Oppenheimer approximation. The field-free basis for 2H-imidazole molecule was produced using the rigid-rotor approximation with the rotational constants calculated from the equilibrium geometry, obtained using density functional theory (DFT) with the B3LYP functional and the def2-QZVPP basis set $[55,56]$. In the second step, the time-dependent solutions for the full molecule-field interaction Hamiltonian were obtained using the computational approach Richmol [57,58]. The wave functions were time-propagated using the splitoperator method with a time step of $10 \mathrm{fs}$. The time-evolution operator was evaluated using an iterative approximation based on the Krylov subspace methods.

\section{RESULTS AND DISCUSSION}

The calculated cumulative population-inversion between $|a\rangle$ and $|c\rangle$ principal rotation states for $\mathrm{D}_{2} \mathrm{~S}$ and $2 \mathrm{H}$-imidazole are shown in Fig. 3; see Fig. 7 in Appendix B for the individual populations. The cumulative population inversion was calculated as the difference between the state populations, in field-free conditions after the 150 ps centrifuge pulse was turned off, summed along the $|a\rangle$ and the $|c\rangle$ excitation paths for $J \geqslant 10$ for $\mathrm{D}_{2} \mathrm{~S}$ and for $J \geqslant 20$ for $2 \mathrm{H}$-imidazole.

As long as the strong ro-vibrational coupling effects do not break the $|a\rangle$ or $|c\rangle$ excitation chain, the final distribution of populations across the states with different $J$ can be controlled by the centrifuge turn-off time. In principle, super-rotor states can be populated. For rigid-rotor $2 \mathrm{H}$-imidazole, the rotational excitation proceeds unhindered up to high angular momentum states. For $\mathrm{D}_{2} \mathrm{~S}$, the $|a\rangle$ rotational-excitation chain breaks around $J=20$ due to the centrifugal distortion effects characteristic for the molecules with rotational energy level clustering [59]. Thus, as a measure of the rotational excitation yield, we use the cumulative populations of states with $J \geqslant 20$ for $2 \mathrm{H}$-imidazole and $J \geqslant 10$ for $\mathrm{D}_{2} \mathrm{~S}$.

The populations plotted in Fig. 3 are functions of the centrifuge peak field $E_{0}$ and the rate of acceleration $\beta$. In both molecules, small acceleration rates populate mainly the $|c\rangle$ 

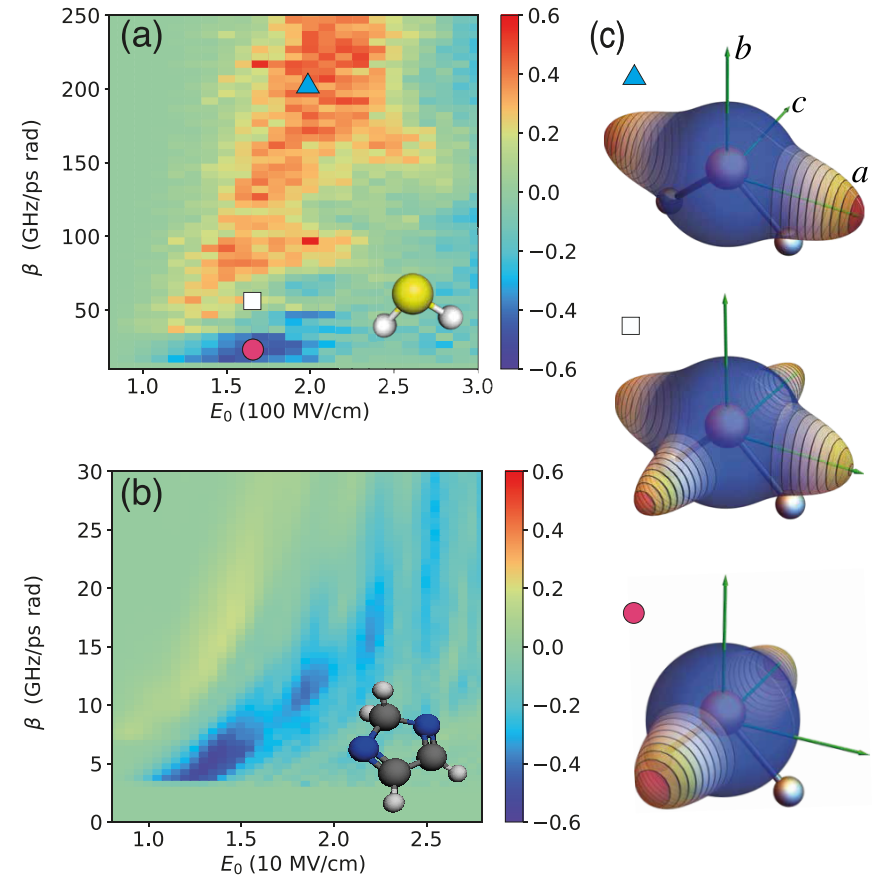

FIG. 3. Differences in cumulative populations of the $|c\rangle$ and $|a\rangle$ principal rotation states as functions of the centrifuge's peak field strength $E_{0}$ and acceleration $\beta$, plotted in (a) for $\mathrm{D}_{2} \mathrm{~S}$ and in (b) for $2 \mathrm{H}$-imidazole; see also Fig. 7. (c) displays $3 \mathrm{D}$ probability distributions for the molecular-frame rotation axes of $\mathrm{D}_{2} \mathrm{~S}$, showing $a, a / c$, and $c$ rotation generated with three different sets of centrifuge parameters, marked in (a) by a triangle, a square, and a circle, respectively.

states. With increasing acceleration rate the optical centrifuge populates more preferably the $|a\rangle$ states. The relation between the wave-packet composition and the probability of the rotation-axis orientation in the molecular frame is displayed in Fig. 3(c) for three selected sets of the centrifuge parameters. A closer inspection of Fig. 3(a) reveals that at small acceleration rates $\beta \approx 50 \mathrm{GHz} / \mathrm{ps}$ solely changing the field from weak to strong switches the created wave packet from $|a\rangle$-dominated to $|c\rangle$-dominated. After initial $|a\rangle /|c\rangle$ bifurcation for $J=0 \rightarrow$ 2 the rotational excitation proceeds nearly loss-free in $\mathrm{D}_{2} \mathrm{~S}$ and, due to its higher density of states, with some losses in 2H-imidazole.

The preference of an asymmetric top molecule to rotate about the $a$ - or $c$ - principal axis of inertia is determined by its polarizability and rotational constants, which define the transition moments between rotational states, and the properties of the centrifuge field. All above quantities can be represented in a vector

$$
S_{i}:=R_{i}\left(Q_{j i}+Q_{k i}\right) \quad i, j, k=a, b, c,
$$

where $i \neq j \neq k \neq i$ label the principal axes of inertia. $R_{i}$ describes the molecule's ability to rotate about an axis $i=$ $a, b, c$ and is defined as $R_{i}=C_{i}\left(\left|\alpha_{j j}-\alpha_{k k}\right|\right) /\left(C_{j}\left(\mid \alpha_{k k}-\right.\right.$ $\left.\left.\alpha_{i i} \mid\right)+C_{k}\left(\left|\alpha_{j j}-\alpha_{i i}\right|\right)\right)$, where $\alpha_{i i}$ are the diagonal components of the electronic polarizability and $C_{i}=a, b, c$ for $i=a, b, c . Q_{i j}$ describes the quantum-mechanical population transferability defined as $Q_{i j}=P_{i} / P_{j}$, with the Landau-Zener populations $P_{i}=1-\exp \left(-\pi \Omega_{i}^{2} /(4 \beta)\right)$ [60] and the Rabi frequency $\Omega_{i}=\mu_{i} E_{0}^{2}$, which depends on the transition moment

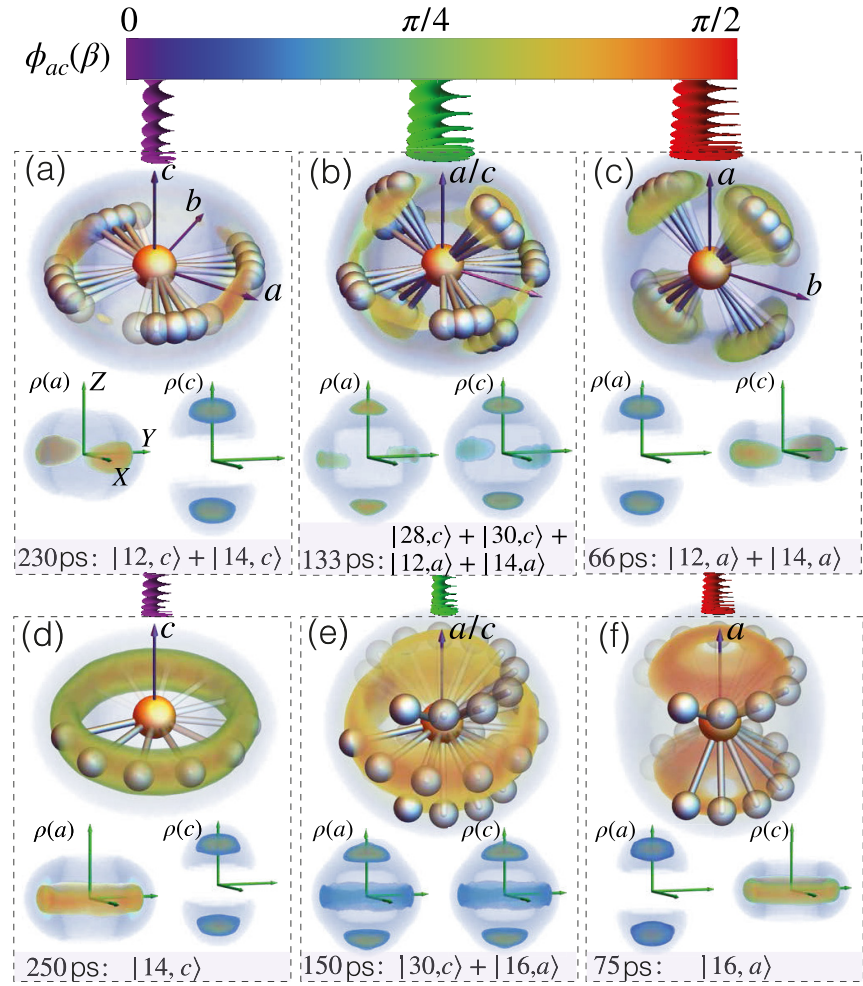

FIG. 4. 3D probability density distributions of the deuterium nuclei and the $a$ and $c$ axis, $\rho(a)$ and $\rho(c)$, of $\mathrm{D}_{2} \mathrm{~S}$. The deuteriumnuclei density plots shown in the upper part of each panel provide a complementary view to the inertial-axis density plots displayed in the panels' bottom part. These distributions are depicted for wave packets generated with an optical centrifuge at three different acceleration rates $\beta=30,115$, and $230 \mathrm{GHz} / \mathrm{ps}$ and field strength $E_{0}=2 \times 10^{8} \mathrm{~V} / \mathrm{cm}$. The different centrifuge-field turnoff times and the resulting wave-packet compositions $|J, a / c\rangle$ are specified below each plot. The colorbar represents values of the angle $\phi_{a c}=\arctan \left(S_{a} / S_{c}\right)$, which correlates with the centrifuge's acceleration rate; see text for details. [(a)-(c)] Wave packets showing different types of 3D alignment; [(d)(f)] wave packets without 3D alignment. The $X Y Z$ coordinate system in (a) indicates the laboratory frame. Relevant principal inertial axes are marked in purple. Blurred atoms reflect the probability density of finding the deuterium and sulfur atoms in 3D space.

from the rotational ground state to one of the $|i\rangle(i=a, b, c)$ principal rotation states and $E_{0}$ is the electric field strength.

The rotability $\vec{S}$ (3) quantifies the molecule's preference to rotate about the different inertial axes. It is composed of the two quantities: (1) the trapability $R_{i}$ measures the molecule's capability to form certain pendular bound-states in a centrifuge field, i.e., it provides a measure of how confined the molecule is in the pendular potential well along each of the principal axes. (2) The quantum transferability $Q_{i j}$ accounts for the transition moment differences between the respective $|a\rangle,|b\rangle,|c\rangle$ principal rotational excitation branches. It quantifies the preference of the system to choose one rotational excitation path over the other, as a function of the optical centrifuge parameters $\beta$ and $E_{0}$, see Appendix $C$ for details. Figure 4 shows the 3D rotational probability density distributions for the deuterium nuclei in $\mathrm{D}_{2} \mathrm{~S}$ for wave 
packets generated by the optical centrifuge at electric field strength $E_{0}=2 \times 10^{8} \mathrm{~V} / \mathrm{cm}$ and three different acceleration rates: $\beta=30,115$, and $230 \mathrm{GHz} / \mathrm{ps}$. The acceleration rates are conveniently encoded in colorbar representing the angle $\phi_{a c}=\arctan \left(S_{a} / S_{c}\right)$ which relates the $a$ and $c$ elements of the rotability vector $\vec{S}$ given in (3). wave packets simulated at $\phi_{a c} \approx 0$, Figs. 4(a) and 4(d), $\phi_{a c} \approx \pi / 4$, Figs. 4(b) and $4(\mathrm{e})$, and $\phi_{a c} \approx \pi / 2$, Figs. 4(c) and 4(f), display strong characteristics of $|c\rangle,|c\rangle+|a\rangle$ and $|a\rangle$ states, respectively; cf. Fig. 1.

At low acceleration rates $\beta$, the rotability vector is dominated by the $S_{c}$ component $\left(\phi_{a c} \approx 0\right)$ and the rotational wave packet mainly consists of $|c\rangle$ states; the deuterium atoms' 3D probability forms a ring shown in Fig. 4(d). With increasing $\beta$ the ratio $S_{a} / S_{c}$ also increases and the wave packet composition smoothly converts to the $|a\rangle$-state dominant $\left(\phi_{a c} \approx \pi / 2\right)$ in Fig. 4(f). To summarize, for a given set of rotational constants, electronic polarizabilities, and corresponding transition dipole moments, the rotability vector depends solely on the acceleration rate of the centrifuge $\beta$ and its electric field strength $E_{0}$. Thus this rotability vector is a very useful quantity for estimating the centrifuge parameters needed to reach a desired $|a\rangle /|c\rangle$ composition of the wave packet without the need for costly quantum-mechanical computations.

In addition, depending on centrifuge's turn-off time shown below each plot in Fig. 4, the end-product wave packet can become dominated by principal rotation states (or mixtures of those) either with a single $J$ value or with $J, J+2$ coherences. In the latter case, the respective probability densities evolve in time (see Appendix D and Ref. [61]). Snapshots of such wave packets are displayed in Figs. 4(a)-4(c). A high degree of 3D alignment is visible in these wave packets. In Ref. [61], we also show simulated velocity-map images which record $D^{+}$ions after Coulomb exploding the molecules-an experiment which can detect and characterize different principal rotation states.

The new protocol for controlling populations of the $|a\rangle$ and $|c\rangle$ rotational states opens an avenue $[14,15,62]$ to $3 \mathrm{D}$ aligning $[16,19,63]$ molecules with either their largest or smallest polarizability axis pointing along the wave vector of the alignment laser (centrifuge). Such $k$ alignment $[14,49]$ is desired in many ultrafast imaging experiments $[13,28,64-66])$ yet so far has not been realized for asymmetric top molecules. Here, we show that the centrifuge-field turn-off time can be used to steer the 3D $k$ alignment of molecules. For example, the wave packet shown in Fig. 4(a) is dominated by a uniform mixture of $\mathrm{D}_{2} \mathrm{~S}|J=12, c\rangle$ and $|J=14, c\rangle$ states, it exhibits classical-like rotation of 3D-localized nuclear probability density [67]. The molecular $a b$ plane is confined in the $X Y$ rotation plane of the optical centrifuge and the $c$ axis aligned along the wave vector $Z$ of the pulse. Ramping up the centrifuge's acceleration rate to a high value of $\beta=$ $230 \mathrm{GHz} / \mathrm{ps}\left(\phi_{a c} \approx \pi / 2\right)$ yields, after $66 \mathrm{ps}$, approximately a $|J=12, a\rangle+|J=14, a\rangle$ rotational wave packet, depicted in Fig. 4(c), where this time the $a$ axis points along the light's wave vector. Interestingly, for an intermediate acceleration rate $\beta=115 \mathrm{GHz} / \mathrm{ps}\left(\phi_{a c} \approx \pi / 4\right)$, a superposition of $3 \mathrm{D}$ aligned states in which the $a$ and $c$ axes are simultaneously pointing along the laboratory $Z$ axis is shown in the middle plot in Fig. 4(b).

\section{SUMMARY}

In summary, we demonstrated that appropriate values of the optical centrifuge's acceleration rate and intensity gauge the rotational wave-packet composition. Essentially arbitrary coherence between the $|a\rangle$ and $|c\rangle$ principal rotation quantum states can be achieved. Through elementary use of an optical centrifuge one can prepare molecular ensembles in the gas phase in which both the laboratory-fixed angular momentum as well as the molecule-fixed angular momentum are robustly controlled. By appropriately choosing the turn-off time of the centrifuge field one can also create wave packets that exhibit classical-like rotation and exhibit high degrees of 3D alignment with the $a$ or $c$ axis pointing along the wave vector of the driving field.

Natural applications of such tailored wave packets, which are typically long-lived [39], are stereodynamics studies [68], e.g., in crossed-molecular-beam or surface-scattering experiments investigating collisional properties, reactive scattering, or stereodynamical control of chemical reactions.

The presented method for coherent control of the rotation axis is expected to work best for light asymmetric near-oblatetop molecules with large electronic polarizability anisotropies $\Delta \alpha_{1}=2 \alpha_{z z}-\alpha_{x x}-\alpha_{y y}$ and $\Delta \alpha_{2}=\alpha_{x x}-\alpha_{y y}$. In the case of symmetric-top molecules with $\Delta \alpha_{2}=0$, it is only possible to populate the lowest-energy rotational excitation path for prolate tops and the highest-energy path for oblate tops. In light molecules, the lower density of rotational states significantly aids the control of the excitation pathway and at the same time is very well suited for the production of very cold molecular beams using the deflection techniques [8]. On the other hand, lighter molecules generally require stronger fields to efficiently excite the Raman transitions, which can lead to ionization depletion.

The proposed method has a tolerance for the fluctuation in the laser intensity of about $20 \%-25 \%$. We point out that our simulations utilized experimental parameters that are within the capabilities of typical present day laser and molecular beam technology.

\section{ACKNOWLEDGMENTS}

We thank Stefanie Kerbstadt for fruitful discussions. This work has been supported by the Deutsche Forschungsgemeinschaft (DFG) through the priority program "Quantum Dynamics in Tailored Intense Fields" (QUTIF, SPP 1840, YA 610/1) and the Cluster of Excellence "Advanced Imaging of Matter" (AIM, EXC 2056, ID 390715994). We acknowledge support by Deutsches Elektronen-Synchrotron DESY, a member of the Helmholtz Association (HGF), and the use of the Maxwell computational resources operated at Deutsches Elektronen-Synchrotron DESY.

\section{APPENDIX A: SIMPLE MODEL OF THE ROTATIONAL-STATE-POPULATION BRANCHING}

To show how the optical centrifuge's acceleration rate and field strength guides the rotational excitation path in near-oblate asymmetric top molecules we build a simple model of three rotational energy levels: $\left|J_{k_{a} k_{c}}, m\right\rangle=$ $\left|0_{00}, 0\right\rangle,\left|2_{20}, 2\right\rangle,\left|2_{02}, 2\right\rangle$. The appropriate energy levels 


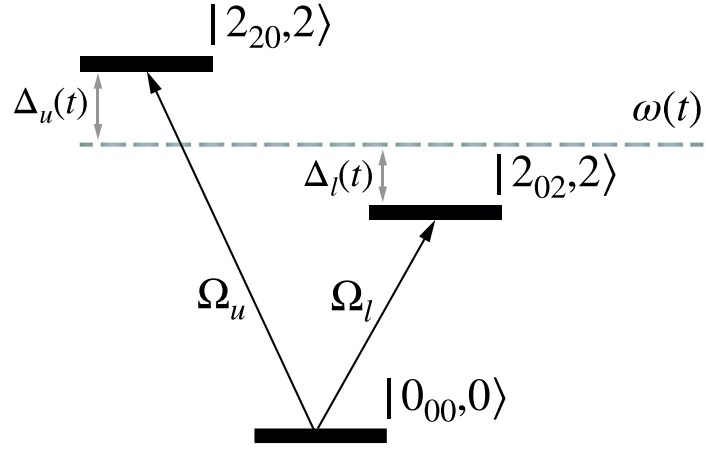

FIG. 5. A model three-level system representing the lowestenergy rotational states in a near-oblate asymmetric top molecule. The levels are denoted as $\left|J_{k_{a} k_{c}}, m\right\rangle=\left|0_{00}, 0\right\rangle,\left|2_{20}, 2\right\rangle,\left|2_{02}, 2\right\rangle$ and the respective Rabi frequencies connecting the levels are shown as $\Omega_{l}, \Omega_{u}$. The dashed horizontal line denotes the instantaneous frequency $\omega(t)$ of the optical centrifuge and appropriate detunings are given as $\Delta_{l}$ and $\Delta_{u}$

together with Rabi frequencies $\Omega_{l}, \Omega_{u}$ connecting these levels are displayed in Fig. 5.

Numerical solutions to the time-dependent Schrödinger equation for this system are shown in Fig. 6 for four different values of the centrifuge's acceleration rate $\beta$; where $\omega(t)=\beta t$. In near-oblate molecules the transition moment to the upper energy level $\left|2_{20}, 2\right\rangle$ dominates over the transition moment to the lower energy level $\left|2_{02}, 2\right\rangle$, see Fig. 2. The ratio of these transition moments depends on the molecule. Here we adopted the ratio $\Omega_{l} / \Omega_{u}=3.0$ observed in $2 \mathrm{H}$-imidazole. The calculated population time-profiles shown in Fig. 6 clearly suggest that at high $\beta$ values the upper energy level is predominantly populated, whereas low acceleration rates of the centrifuge field prefer the lower energy level. Intermediate $\beta$ values create a coherent mixture of both excited states.

\section{APPENDIX B: CUMULATIVE POPULATIONS}

In Fig. 7, we show results of quantum-mechanical calculations of rotational dynamics of $\mathrm{D}_{2} \mathrm{~S}$ and $2 \mathrm{H}$-imidazole
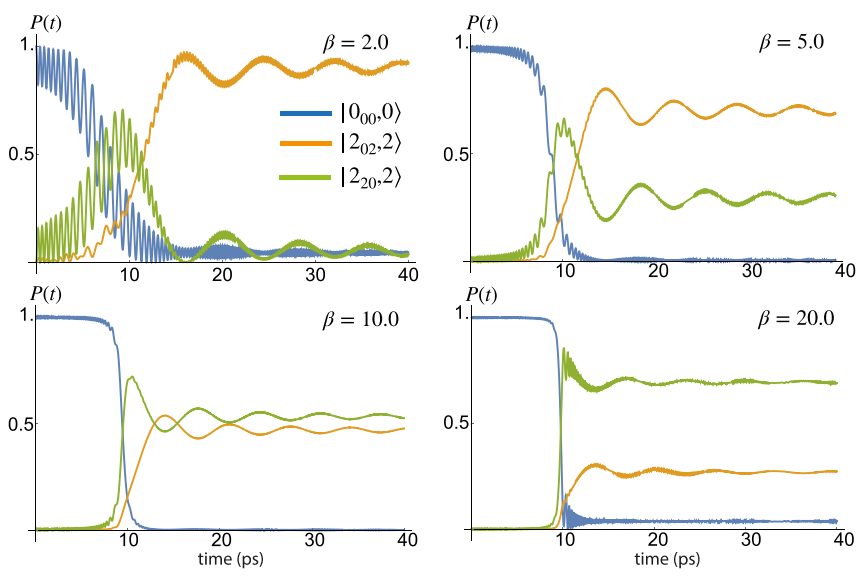

FIG. 6. Calculated population time profiles for the three-level system defined in Fig. 5 at different values of the centrifuge's acceleration rate $\beta=2.0,5.0,10.0,20.0$ (arb. unit). in the optical centrifuge. The calculated cumulative populations of the $|a\rangle$ and $|c\rangle$ principal rotation states are shown in Figs. 7(a)-7(f). These were calculated for $J \geqslant 10$ for $D_{2} S$ and $J \geqslant 20$ for $2 \mathrm{H}$-imidazole in field-free conditions after the 150 ps centrifuge pulse was turned off. The populations are plotted as functions of the centrifuge peak field $E_{0}$ and the rate of acceleration $\beta$. Figures 7(c) and 7(f) show the population inversion between $|a\rangle$ and $|c\rangle$ states. In both molecules, small acceleration rates populate mainly the $|c\rangle$ states. With increasing acceleration rate the optical centrifuge populates more preferably the $|a\rangle$ states. The relation between the wavepacket composition and the probability of the rotation-axis orientation in the molecular frame is displayed in Fig. 7(g) for three selected sets of the centrifuge parameters. The lower overall excitation efficiency in $2 \mathrm{H}$-imidazole than in $\mathrm{D}_{2} \mathrm{~S}$ is caused by its higher density of states, already at small $J=2$ and 4 values, which results in further bifurcations and eventually losses from the centrifuge.

\section{APPENDIX C: MOLECULAR ROTABILITY}

The purpose for the molecular rotability introduced in (3) is to qualitatively characterize the relation between the optical centrifuge field parameters $\left(\beta, E_{0}\right)$ and those of the molecule, the polarizability tensor $\alpha_{i j}$, moments of inertia tensor $I_{i}$, and dipole transition moments $\mu_{k l}$ with the composition of the rotational wave packet.

A linear molecule in the optical centrifuge field follows the classical equation of motion $I \ddot{\theta}=-\beta I-U_{0} \sin 2 \theta$, where $U_{0}=\frac{1}{4} E_{0}\left(\alpha_{\|}-\alpha_{\perp}\right)$ is the pendular potential depth. Here $\alpha_{\|}$ is the electronic polarizability along the long molecular axis and $\alpha_{\perp}$ is the perpendicular component of the polarizabiilty. $I$ is the moment of inertia and $\theta$ denotes the angle between the molecular long axis and the electric field vector of length $E_{0}$.

In order to capture the likelihood of trapping the molecule in the pendular potential well created by the centrifuge field one needs to compare the angular acceleration of the centrifuge field with the angular acceleration of the molecule caused by this field, as shown below:

$$
\beta<\frac{K_{\|, \max }}{I}=-\frac{\left.\nabla_{\theta} V(\theta)\right|_{\max }}{I} \approx \frac{U_{0}}{I},
$$

where $V(\theta)$ is the pendular potential and $K_{\|, \max }$ is the maximum torque applied to the molecule. The trapping condition given in $(\mathrm{C} 1)$ can be also directly inferred from the classical equation of motion: $\frac{\ddot{\theta}+\beta}{\beta}=-\frac{U_{0}}{I \beta} \sin 2 \theta$. Minding the condition in (C1), a single parameter which quantifies the trapability of a linear molecule in the centrifuge field can be written as

$$
S^{(1 \mathrm{D})}:=\frac{E_{0}^{2}}{\beta} \frac{\alpha_{\|}-\alpha_{\perp}}{I} .
$$

If the acceleration $\beta$ of the field is lower than the acceleration (response) of the molecule in this field, the molecule is effectively trapped in the pendular potential well and becomes centrifuged, i.e., rotationally excited. Thus, for $S^{(1 D)} \gg 1$, the rotational excitation should be efficient.

What distinguishes asymmetric-top molecules from linear molecules is that in the former there is more than one possible rotational excitation pathway. In linear molecules, the angular momentum is always perpendicular to the molecule's 

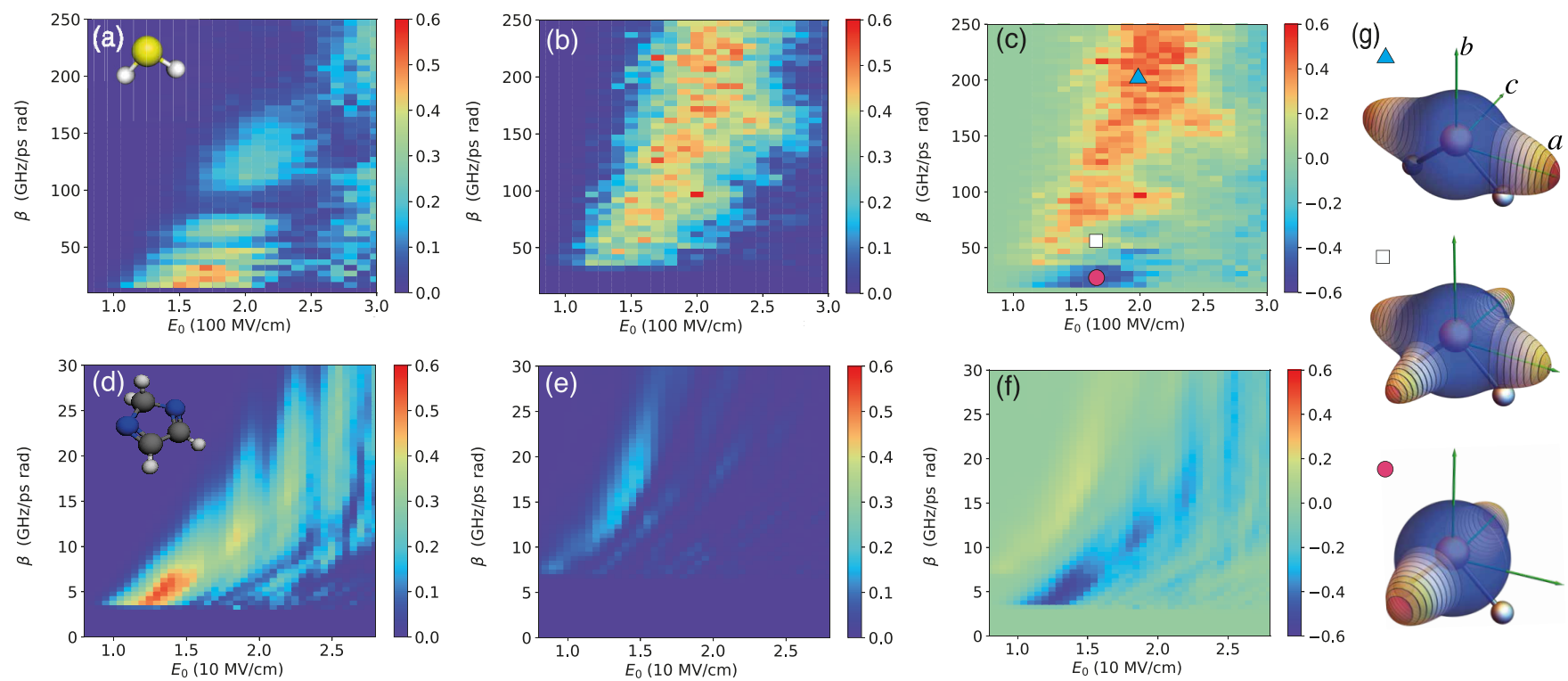

FIG. 7. Cumulative populations of the $|c\rangle$ and $|a\rangle$ principal rotation states as functions of the centrifuge's peak field strength $E_{0}$ and acceleration $\beta$, plotted for $\mathrm{D}_{2} \mathrm{~S}$ in (a) and (b), respectively, and for $2 \mathrm{H}$-imidazole in (d) and (e). Corresponding cumulative population differences between $|c\rangle$ and $|a\rangle$ states are plotted in (c) and (f). For clarity, these populations were only plotted for $J \geqslant 10$ for $\mathrm{D}_{2} \mathrm{~S}$ and for $J \geqslant 20$ for $2 \mathrm{H}$-imidazole. (g) $3 \mathrm{D}$ probability distributions for the molecular-frame rotation axes of $\mathrm{D}_{2} \mathrm{~S}$ are showing $a, a / c$, and $c$ rotation generated with three different sets of centrifuge parameters, marked in panel (c) by a triangle, a square, and a circle, respectively.

long axis. In asymmetric-top molecules the excitation can be accompanied by a change in the molecular-frame angular momentum along the $a, b$, or $c$ principal axis. Therefore the 3D-rotability measure must distinguish rotational excitations

along the different principal inertia axes. For this reason, a generalization of (C2) to asymmetric-top molecules must capture the details of the full 3D molecule-field-interaction potential given by

$$
\begin{aligned}
V(\theta, \phi, \chi, t)= & \frac{1}{4} \epsilon_{0}^{2} \cos ^{2}(\omega t)\left[-\frac{2}{3}\left(\alpha_{x x}+\alpha_{y y}+\alpha_{z z}\right) D_{00}^{(0) *}-e^{2 i \beta t^{2}}\left(\alpha_{x z}-i \alpha_{y z}\right) D_{-2,-1}^{(2) *}-e^{-2 i \beta t^{2}}\left(\alpha_{x z}-i \alpha_{y z}\right) D_{2,-1}^{(2) *}+\right. \\
& +e^{2 i \beta t^{2}}\left(\alpha_{x z}+i \alpha_{y z}\right) D_{-2,1}^{(2) *}+e^{-2 i \beta t^{2}}\left(\alpha_{x z}+i \alpha_{y z}\right) D_{2,1}^{(2) *}+\sqrt{\frac{2}{3}}\left(\alpha_{x z}-i \alpha_{y z}\right) D_{0,-1}^{(2) *} \sqrt{\frac{2}{3}}\left(\alpha_{x z}+i \alpha_{y z}\right) D_{0,1}^{(2) *}+ \\
& +\frac{1}{\sqrt{6}} e^{2 i \beta t^{2}}\left(\alpha_{x x}+\alpha_{y y}-2 \alpha_{z z}\right) D_{-2,0}^{(2) *}+\frac{1}{\sqrt{6}} e^{-2 i \beta t^{2}}\left(\alpha_{x x}+\alpha_{y y}-2 \alpha_{z z}\right) D_{2,0}^{(2) *}-\frac{1}{3}\left(\alpha_{x x}+\alpha_{y y}-2 \alpha_{z z}\right) D_{0,0}^{(2) *} \\
& -\frac{1}{2} e^{2 i \beta t^{2}}\left(\alpha_{x x}-2 i \alpha_{x y}-\alpha_{y y}\right) D_{-2,-2}^{(2) *}-\frac{1}{2} e^{2 i \beta t^{2}}\left(\alpha_{x x}+2 i \alpha_{x y}-\alpha_{y y}\right) D_{-2,2}^{(2) *}+\frac{1}{\sqrt{6}}\left(\alpha_{x x}-2 i \alpha_{x y}-\alpha_{y y}\right) D_{0,-2}^{(2) *}+ \\
& \left.+\frac{1}{\sqrt{6}}\left(\alpha_{x x}+2 i \alpha_{x y}-\alpha_{y y}\right) D_{0,2}^{(2) *}-\frac{1}{2} e^{-2 i \beta t^{2}}\left(\alpha_{x x}-2 i \alpha_{x y}-\alpha_{y y}\right) D_{2,-2}^{(2) *}-\frac{1}{2} e^{-2 i \beta t^{2}}\left(\alpha_{x x}+2 i \alpha_{x y}-\alpha_{y y}\right) D_{2,2}^{(2) *}\right]
\end{aligned}
$$

where $D_{\Delta M, \Delta k}^{(J) *}$ is the complex-conjugated Wigner $D$ matrix and $\theta, \phi, \chi$ are the Euler angles. The elements of static polarizability tensor $\alpha_{x x}, \alpha_{x y}, \alpha_{x z}, \alpha_{y y}, \alpha_{y z}$, and $\alpha_{z z}$ refer to the molecule-fixed principal-axis-of-inertia frame. Accordingly, the 3D molecular rotability must be a vector with its three components denoting the net affinity of the molecule to rotate about the three-respective principal axes as a function of the centrifuge field parameters. In order to heuristically derive the components of the 3D molecular rotability, we follow the condition given in $(\mathrm{C} 1)$ and calculate the torques created along the respective principal inertia axes.

Here we note that rather than deriving a condition for the molecule to stay in the centrifuge potential energy trap, we aim at providing a measure, which weighs the relative affinity of the principal polarisability axes to the polarization plane of the centrifuge, i.e., which of the principal rotation axes is the most likely. Without referring to complex classical dynamics of the rigid $3 \mathrm{D}$ molecule in rotating electric field we give below a simplified justification for molecular rotability. 
First note that for each of three possible axes of rotation there is an effective torque caused by the interaction with the cenitrfuge field. The relative magnitude of the torque $K_{\|}^{(\lambda)}$ created along the $\lambda$ axis (aligned with $Z$ axis) to the torque $K_{\perp}^{(\lambda)}$ along an axis perpendicular to $Z$ (in the $X Y$ plane) informs about the affinity of the molecule to stay aligned along the given rotation axis. We imagine three arrangements, in which the molecule rotates about the $a, b$, or $c$ axis aligned along the laboratory $Z$ axis and calculate the ratio of the aforementioned torques:

$$
\xi_{\lambda}=\frac{K_{\|}^{(\lambda)}}{K_{\perp}^{(\lambda)}}, \quad \lambda=a, b, c
$$

with

$$
\begin{aligned}
& K_{\|}^{(\lambda)}=\left|\frac{\partial V(t)}{\partial \phi}\right|, \\
& K_{\perp}^{(\lambda)}=\left|\frac{\partial V(t)}{\partial \theta}\right|,
\end{aligned}
$$

where $V_{i}(t)$ denotes the potential given in (C3) and $\theta, \phi$ are the azimuthal and polar Euler angles, respectively. With (C3) the parallel and perpendicular torque components are given as

$$
\begin{aligned}
& K_{\|}^{(\lambda)}=\frac{\epsilon_{0}^{2}}{8}\left|\left(\alpha_{x x}-\alpha_{y y}\right) d_{-2,2}^{(2)}(\theta)\right|, \\
& K_{\perp}^{(\lambda)}=\frac{\epsilon_{0}^{2}}{4 \sqrt{6}}\left|\left(\left(\alpha_{x x}-\alpha_{z z}\right)+\left(\alpha_{y y}-\alpha_{z z}\right)\right) \frac{\partial d_{-2,0}^{(2)}(\theta)}{\partial \theta}\right|,
\end{aligned}
$$

where $d_{\Delta m, \Delta k}^{(J)}$ are elements of the real-valued Wigner small-d matrix. We dropped any contributions from the off-diagonal elements of the electronic polarizability tensor. In majority of small molecules the principal inertia axes frame and the frame in which the electronic polarizability is diagonal nearly overlap, which means that the off-diagonal elements of the electronic polarizability in the principal inertia axes frame are very small compared to the diagonal elements. We note that $d_{-2,0}^{(2)}(\theta)=\sqrt{\frac{3}{8}} \sin ^{2} \theta$ and $d_{-2,-2}^{(2)}(\theta)=\cos ^{4} \theta / 2$ and are bound from above by 1 and $\sqrt{\frac{3}{8}}$, respectively. Therefore the ratio of maximum torques is approximately given as

$$
\xi_{\lambda} \approx \frac{\left|\alpha_{x x}-\alpha_{y y}\right|}{\left|\left(\alpha_{x x}-\alpha_{z z}\right)+\left(\alpha_{y y}-\alpha_{z z}\right)\right|},
$$

where $\lambda=a, b, c$. Based on (C7) we propose a definition for the generalized trapability (trapping ability), which accounts for these maximum torques, in the following form:

$$
R_{i}=\frac{C_{i}\left|\alpha_{j j}-\alpha_{k k}\right|}{C_{j}\left|\alpha_{k k}-\alpha_{i i}\right|+C_{k}\left|\alpha_{j j}-\alpha_{i i}\right|}, \quad i=a, b, c .
$$

This definition comprises the relative pendular potential depths projected along different principal inertia axes. Here, $\alpha_{i i}$ are the diagonal components of the electronic polarizability and $C_{i}=a, b, c$ for $i=a, b, c$ are the rotational constants. In simple terms the, 3D trapability is constructed from 1D trapabilities and reflects the molecule's affinity to rotate about a given inertial axis as compared to the other two axes. We assume that the principal axes of inertia system is parallel to the electronic polarizability axis system, which is a very good approximation for $\mathrm{D}_{2} \mathrm{~S}$ and $2 \mathrm{H}$-imidazole.
Another factor that contributes to the final populations of rotational states upon rotational excitation with the optical centrifuge are the transition-dipole moments to the upper and the lower rotational excitation branches. The interaction of molecules with nonresonant chirped fields such as the optical centrifuge can be qualitatively described with the Landau-Zener model [60]. The branching ratio for the rotational excitation along the upper or lower excitation path, see Fig. 1, can be estimated as the ratio of the Landau-Zener populations:

$$
Q_{i j}=P_{i} / P_{j},
$$

where $P_{i}=1-\exp \left(-\pi \Omega_{i}^{2} /(4 \beta)\right) \quad$ [60] and the Rabi frequency $\Omega_{i}=\mu_{i} E_{0}^{2}$, which depends on the transition moment $\mu_{i}$ from the rotational ground state to one of the $|i\rangle(i=$ $a, b, c)$ principal rotation states and $E_{0}$ is the electric field strength. The quantum-mechanical population transferability $Q_{i j}$ relates the centrifuge field parameters to the anticipated branching ratio for the population transfer to respective rotational states.

Putting together the molecular trapability and transferability we can assume that the molecular trapability given in (C8) serves as a scaling factor for the rotational excitation probability to the upper-energy-level branch $(|a\rangle$ states) versus the lower-energy-levels branch ( $|c\rangle$ states), quantified by the trasferability $Q_{i j}$.

Finally the 3D molecular rotability can be written as

$$
S_{i}:=R_{i}\left(Q_{j i}+Q_{k i}\right) \quad i, j, k=a, b, c .
$$

In the prolate $(A>B=C)$ and oblate $(A=B>C)$ symmetric-top limits, the rotability vector becomes

$$
\begin{gathered}
\vec{S}_{\text {prolate }} \rightarrow\left(\begin{array}{l}
0 \\
Q \\
Q
\end{array}\right), \\
\vec{S}_{\text {oblate }} \rightarrow\left(\begin{array}{l}
Q \\
Q \\
0
\end{array}\right),
\end{gathered}
$$

where $Q=Q_{b a}+Q_{c a}$. Thus, in prolate (oblate) symmetric tops there is no possibility for creating torque in the $b c(a b)$ molecular plane, i.e., the probability for rotational excitation about the $a(c)$ axis through the electronic polarizability is 0 . In Fig. 8, we show an example of the molecular rotability components $S_{a}-S_{c}$ for the molecular parameters of $2 \mathrm{H}$-imidazole as a function of the centrifuge electric field strength $E_{0}$ and acceleration rate $\beta$. We see that at low acceleration rates the $S_{c}$ component dominates over the $S_{a}$ component, which suggests that the molecule will most likely follow the $c$ axis rotational excitation branch. The rotability measure covers all wave packets, thus the rotational wave packet can be expressed in terms of the rotability vector components:

$$
|\psi\rangle=\frac{2}{\pi}\left(\arctan ^{2}\left(\frac{S_{a}}{S_{c}}\right)|a\rangle+\sqrt{1-\arctan ^{2}\left(\frac{S_{a}}{S_{c}}\right)}|c\rangle\right)
$$

Geometrically, (C13) represents a map between the combined molecular parameters and the parameters of the centrifuge 
(a)

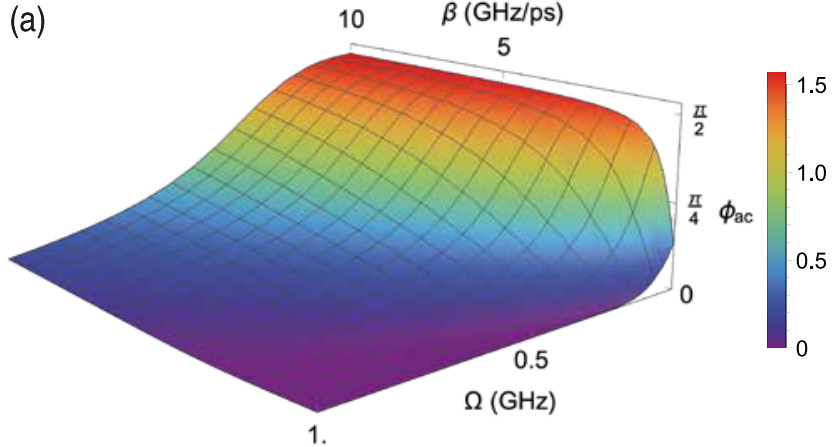

(b)

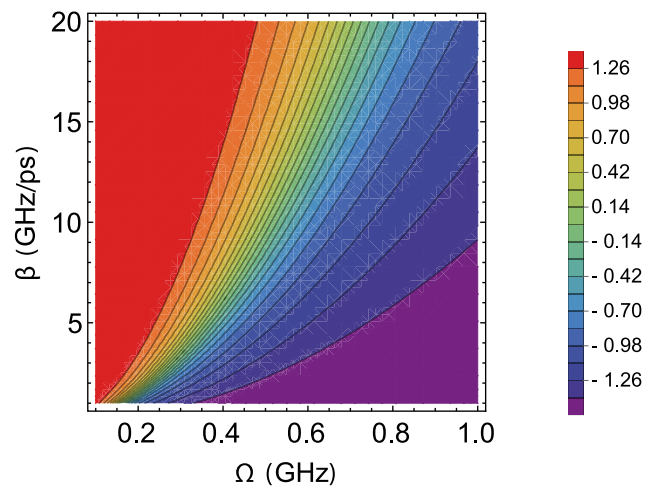

FIG. 8. (a) Graphical representation of the relation between the molecular rotability components $S_{a}$ and $S_{c}$ in the form of the angle: $\phi_{a c}=\arctan \left(\frac{S_{a}}{S_{c}}\right) . \beta$ is the centrifuge acceleration rate and $\Omega$ is the Rabi frequency for a unit transition dipole moment. (b) Molecular rotability map with predicted population inversion between the $a$ and $c$ rotational-excitation branch. The plotted function is $\arctan \left(\frac{S_{a}}{S_{c}}-\frac{S_{c}}{S_{a}}\right)$.

onto a line connecting $|a\rangle$ and $|c\rangle$ states. Rotational states can be classified according to the respective average values of the molecule-fixed angular momentum, and can be represented on a triangle, as shown in the inset of Fig. 1. Vertices of the triangle denote essentially pure $|a\rangle,|b\rangle,|c\rangle$ states. In principle, the composition of the final rotational wave packet created by the interaction with external fields can be mapped onto this triangle. However, because the rotational excitation about the intermediate $b$ axis is very improbable, due to low values of appropriate transition moments, the molecular rotability practically maps the space of centrifuge's parameters $E_{0}$ and $\beta$ onto the edge of the rotational states triangle connecting the $|a\rangle$ and $|c\rangle$ vertices. For this reason, the image of the molecular rotability map for the optical centrifuge is approximately onedimensional.

Figure $8(\mathrm{~b})$ reflects the magnitude of population inversion in the rotational $a$ or $c$ branches. It shows a qualitative agreement with the full quantum-mechanical optical centrifuge calculations for $2 \mathrm{H}$-imidazole, shown in Fig. 3.

\section{APPENDIX D: ANALYSIS OF ROTATIONAL WAVE PACKETS FOR $\mathrm{D}_{2} \mathrm{~S}$ : PROBABILITY DENSITIES AND ALIGNMENT COSINES}

The approximate wave packets generated with an optical centrifuge at three different acceleration rates (a)
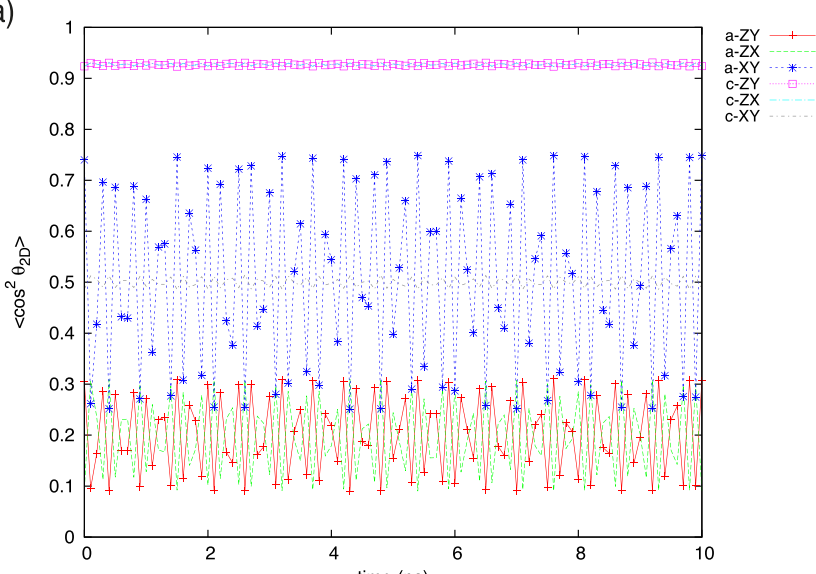

(b)
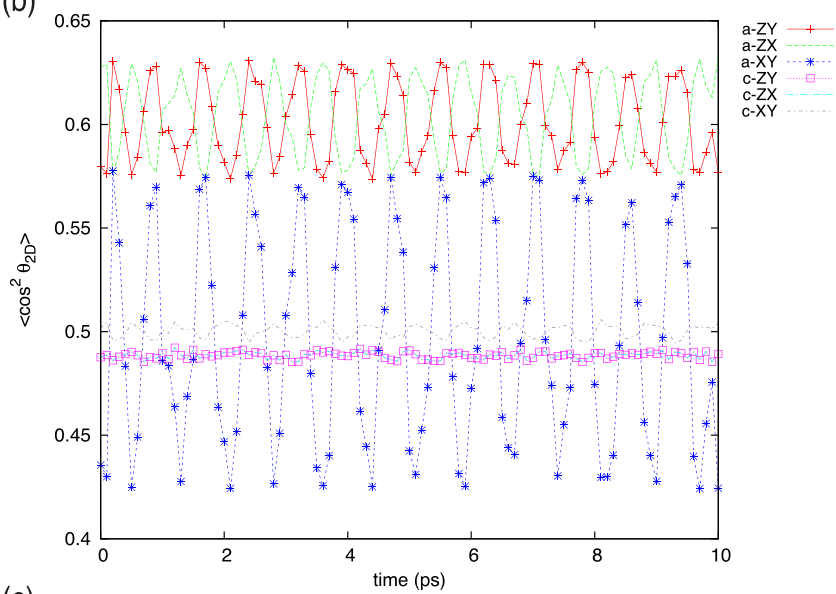

(c)

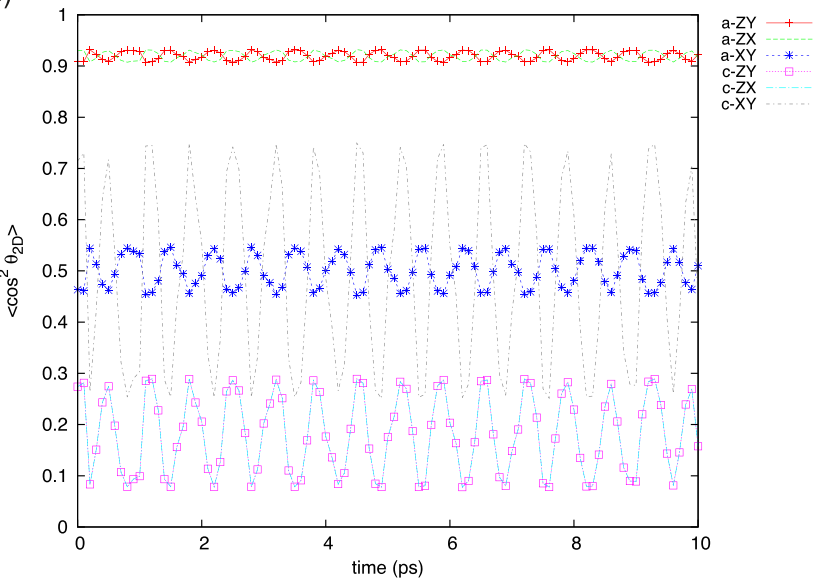

FIG. 9. Calculated 2D alignment-cosine time-profiles for wave packets generated with an optical centrifuge with field strength $E_{0}=2 \times 10^{8} \mathrm{~V} / \mathrm{cm}$ and three different acceleration rates [(a)-(c)] $\beta=30,115$, and $230 \mathrm{GHz} / \mathrm{ps}$, respectively. The centrifuge-field release times were 228, 133, and 66 ps, respectively, see Fig. 4 . The alignment cosines measure the degree of alignment of the principal axes of inertia of $D_{2} S$ with respect to the laboratory axes defined by the centrifuge field. For instance, $a-Z Y$ is the $2 \mathrm{D}$ alignment cosine calculated with respect to the laboratory $Z$ axis of the projection of the molecular $a$ axis onto the $Z Y$ detector plane.

$\beta=30,115$, and $230 \mathrm{GHz} / \mathrm{ps}$ and field strength $E_{0}=2 \times$ $10^{8} \mathrm{~V} / \mathrm{cm}$, shown in Fig. 4, are given as

$$
|\psi\rangle_{\beta=30} \sim 0.55 e^{-i \omega_{12 a} t}|12, a\rangle+0.55 e^{-i \omega_{14 a} t}|14, a\rangle,
$$




$$
\begin{aligned}
|\psi\rangle_{\beta=115} \sim & 0.32 e^{-i \omega_{28 c} t}|28, c\rangle+0.32 e^{-i \omega_{30 c} t}|30, c\rangle \\
+ & 0.32 e^{-i \omega_{2 c} t}|2, c\rangle+0.61 e^{-i \omega_{14 a} t}|14, a\rangle, \\
|\psi\rangle_{\beta=230} \sim & 0.36 e^{-i \omega_{12 c} t}|12, c\rangle+0.36 e^{-i \omega_{14 c} t}|14, c\rangle,
\end{aligned}
$$

where $|J, a / c\rangle$ are the asymmetric top wavefunctions with total angular momentum $J$ and the molecular-frame angular momentum nearly aligned with $a$ or $c$ axis, respectively. All states have $M=J$, i.e., the laboratory-frame angular momentum is aligned along the $Z$ axis.

Time-evolution of the rotation-axis 3D probability $\left(1-e^{-1}\right.$ cutoff) in the above wave packets is displayed in the animated movie files attached [61]: D2S- $\beta$-a-axis.mov, D2S- $\beta$-b-axis.mov, D2S- $\beta$-c-axis.mov for the $a$, $b$ and $c$ principal inertia axes, respectively. Joint 3D probability plots for the deuterium nuclei are given in files D2S- $\beta$-D-atoms.mov. In these movie files, a classical-like rotation of the probability is visible for all three wave packets given in (D1)-(D3).

These rotational wave packets can be straightforwardly detected with the use of velocity-map imaging (VMI) of fragments produced through multiple ionization by ultrashort laser pulses followed by Coulomb explosion $[69,70]$. Files named D2S- $\beta$-vmixz.mov, D2S- $\beta$-vmiyz.mov and D2S- $\beta$-vmixy . mov (see Ref. [61]) present time-evolution of the VMI images simulated for the deuterium ion fragments, assuming axial recoil. The detector is placed in the laboratoryfixed $X Z, Y Z$, and $X Y$ planes, respectively, where $Z$ is the laser propagation direction. Only the first two setups $(X Z$, $Y Z$ ) are routinely implemented in the experiment. The VMI images can be quantitatively characterized by calculating the degree of alignment of selected atoms or principal axes of inertia with respect to the laboratory $X, Y, Z$ axes. The alignment cosine values, which reconstruct the experimental VMI images, can be calculated from the positions of selected atoms or molecular axes $x, y, z$ in the laboratory-frame $X, Y, Z$ using

$$
\left\langle\cos ^{2} \theta_{2 \mathrm{D}}\right\rangle=\left\langle\psi\left|\frac{Z^{2}}{X^{2}+Z^{2}}\right| \psi\right\rangle,
$$

where $\theta_{2 D}$ is an angle in the detector plane $X Z$ between the detector's $Z$ axis and the D-S bond in $\mathrm{D}_{2} \mathrm{~S}$ at the time of the Coulomb explosion. In this work, the alignment cosine values were calculated with Monte Carlo sampling, using $10^{6}$ sampling points, of the rotational wavefunction $|\psi\rangle$ at a given time. Figure 9 displays values of $\left\langle\cos ^{2} \theta_{2 \mathrm{D}}\right\rangle$ calculated for the vectors pointing along the molecular $a$ and $c$ axis, respectively, relative to the laboratory-fixed planes: $X Y, X Z$, and $Y Z$.

Figure 9 shows a high $(>0.9)$ degree of permanent alignment of the $c$ axis along the laboratory fixed $Z$ axis for the wave packet given in (D1) generated with $\beta=30 \mathrm{GHz} / \mathrm{ps}$ and field strength $E_{0}=2 \times 10^{8} \mathrm{~V} / \mathrm{cm}$, see Fig. 4. The classicallike rotational motion of the $a b$ molecular plane about the $c$ axis is reflected in the oscillations of the blue line $(a-X Y)$ in Fig. 9(a). Small amplitude oscillations of the red and green $a-Z Y$ and $a-Z X$ lines near $\left\langle\cos ^{2} \theta_{2 \mathrm{D}}\right\rangle=0.2$ suggest slight nutation of the $a$ axis out of the $X Y$ plane in the laboratory frame. Wave packets shown in (D1)-(D3) exhibit persistent $3 \mathrm{D}$ alignment of the molecule in the frame rotating with the frequency of the coherent classical-like rotation of the whole wave packet. Period of this uniform rotation is in the order of few picoseconds, which is long enough for most of imaging ultrafast dynamics experiments.
[1] P. R. Brooks, Reactions of oriented molecules, Science 193, 11 (1976).

[2] B. Friedrich and D. Herschbach, Alignment and Trapping of Molecules in Intense Laser Fields, Phys. Rev. Lett. 74, 4623 (1995).

[3] H. Stapelfeldt and T. Seideman, Colloquium: Aligning molecules with strong laser pulses, Rev. Mod. Phys. 75, 543 (2003).

[4] C. P. Koch, M. Lemeshko, and D. Sugny, Quantum control of molecular rotation, Rev. Mod. Phys. 91, 035005 (2019).

[5] Cold Molecules: Theory, Experiment, Applications, edited by R. Krems, W. C. Stwalley, and B. Friedrich (CRC Press, Taylor \& Francis Group, Boca Raton, 2009).

[6] L. Holmegaard, J. H. Nielsen, I. Nevo, H. Stapelfeldt, F. Filsinger, J. Küpper, and G. Meijer, Laser-Induced Alignment and Orientation of Quantum-State-Selected Large Molecules, Phys. Rev. Lett. 102, 023001 (2009).

[7] O. Ghafur, A. Rouzée, A. Gijsbertsen, W. K. Siu, S. Stolte, and M. J. J. Vrakking, Impulsive orientation and alignment of quantum-state-selected NO molecules, Nat. Phys. 5, 289 (2009).

[8] Y.-P. Chang, D. A. Horke, S. Trippel, and J. Küpper, Spatiallycontrolled complex molecules and their applications, Int. Rev. Phys. Chem. 34, 557 (2015).
[9] F. Rosca-Pruna and M. J. J. Vrakking, Experimental Observation of Revival Structures in Picosecond LaserInduced Alignment of $\mathrm{I}_{2}$, Phys. Rev. Lett. 87, 153902 (2001).

[10] A. Chatterley, E. T. Karamatskos, C. Schouder, L. Christiansen, A. V. Jörgensen, T. Mullins, J. Küpper, and H. Stapelfeldt, Switched wave packets with spectrally truncated chirped pulses, J. Chem. Phys. 148, 221105 (2018).

[11] E. T. Karamatskos, S. Raabe, T. Mullins, A. Trabattoni, P. Stammer, G. Goldsztejn, R. R. Johansen, K. Długołęcki, H. Stapelfeldt, M. J. J. Vrakking, S. Trippel, A. Rouzée, and J. Küpper, Molecular movie of ultrafast coherent rotational dynamics of OCS, Nat. Commun. 10, 3364 (2019).

[12] S. Fleischer, Y. Zhou, R. W. Field, and K. A. Nelson, Molecular Orientation and Alignment by Intense Single-Cycle THz Pulses, Phys. Rev. Lett. 107, 163603 (2011).

[13] C. T. L. Smeenk, L. Arissian, A. V. Sokolov, M. Spanner, K. F. Lee, A. Staudte, D. M. Villeneuve, and P. B. Corkum, Alignment Dependent Enhancement of the Photoelectron Cutoff for Multiphoton Ionization of Molecules, Phys. Rev. Lett. 112, 253001 (2014).

[14] C. T. L. Smeenk and P. B. Corkum, Molecular alignment using circularly polarized laser pulses, J. Phys. B 46, 201001 (2013). 
[15] A. Korobenko and V. Milner, Adiabatic Field-Free Alignment of Asymmetric Top Molecules with an Optical Centrifuge, Phys. Rev. Lett. 116, 183001 (2016).

[16] J. J. Larsen, K. Hald, N. Bjerre, H. Stapelfeldt, and T. Seideman, Three Dimensional Alignment of Molecules Using Elliptically Polarized Laser Fields, Phys. Rev. Lett. 85, 2470 (2000).

[17] H. Tanji, S. Minemoto, and H. Sakai, Three-dimensional molecular orientation with combined electrostatic and elliptically polarized laser fields, Phys. Rev. A 72, 063401 (2005).

[18] A. Rouzee, S. Guerin, O. Faucher, and B. Lavorel, Field-free molecular alignment of asymmetric top molecules using elliptically polarized laser pulses, Phys. Rev. A 77, 043412 (2008).

[19] I. Nevo, L. Holmegaard, J. H. Nielsen, J. L. Hansen, H. Stapelfeldt, F. Filsinger, G. Meijer, and J. Küpper, Laserinduced 3D alignment and orientation of quantum state-selected molecules, Phys. Chem. Chem. Phys. 11, 9912 (2009).

[20] K. Lin, I. Tutunnikov, J. Qiang, J. Ma, Q. Song, Q. Ji, W. Zhang, H. Li, F. Sun, X. Gong, H. Li, P. Lu, H. Zeng, Y. Prior, I. S. Averbukh, and J. Wu, All-optical field-free three-dimensional orientation of asymmetric-top molecules, Nat. Commun. 9, 5134 (2018).

[21] S. Zhdanovich, A. A. Milner, C. Bloomquist, J. Floß, I. Sh. Averbukh, J. W. Hepburn, and V. Milner, Control of Molecular Rotation with a Chiral Train of Ultrashort Pulses, Phys. Rev. Lett. 107, 243004 (2011).

[22] V. Milner and J. W. Hepburn, Laser control of ultrafast molecular rotation, Adv. Chem. Phys. 159, 395 (2016).

[23] A. A. Milner, A. Korobenko, and V. Milner, Field-free longlived alignment of molecules with a two-dimensional optical centrifuge, Phys. Rev. A 93, 053408 (2016).

[24] A. Owens, A. Yachmenev, S. N. Yurchenko, and J. Küpper, Climbing the Rotational Ladder to Chirality, Phys. Rev. Lett. 121, 193201 (2018)

[25] F. Filsinger, G. Meijer, H. Stapelfeldt, H. Chapman, and J. Küpper, State- and conformer-selected beams of aligned and oriented molecules for ultrafast diffraction studies, Phys. Chem. Chem. Phys. 13, 2076 (2011).

[26] J. Itatani, J. Levesque, D. Zeidler, H. Niikura, H. Pépin, J. C. Kieffer, P. B. Corkum, and D. M. Villeneuve, Tomographic imaging of molecular orbitals, Nature (London) 432 , 867 (2004).

[27] M. Meckel, D. Comtois, D. Zeidler, A. Staudte, D. Pavičić, H. C. Bandulet, H. Pépin, J. C. Kieffer, R. Dörner, D. M. Villeneuve, and P. B. Corkum, Laser-induced electron tunneling and diffraction, Science 320, 1478 (2008).

[28] L. Holmegaard, J. L. Hansen, L. Kalhøj, S. L. Kragh, H. Stapelfeldt, F. Filsinger, J. Küpper, G. Meijer, D. Dimitrovski, M. Abu-samha, C. P. J. Martiny, and L. B. Madsen, Photoelectron angular distributions from strong-field ionization of oriented molecules, Nat. Phys. 6, 428 (2010).

[29] C. J. Hensley, J. Yang, and M. Centurion, Imaging of Isolated Molecules with Ultrafast Electron Pulses, Phys. Rev. Lett. 109, 133202 (2012).

[30] A. Barty, J. Küpper, and H. N. Chapman, Molecular imaging using X-ray free-electron lasers, Annu. Rev. Phys. Chem. 64, 415 (2013)

[31] J. Küpper, S. Stern, L. Holmegaard, F. Filsinger, A. Rouzée, A. Rudenko, P. Johnsson, A. V. Martin, M. Adolph, A. Aquila, S. Bajt, A. Barty, C. Bostedt, J. Bozek, C. Caleman, R. Coffee, N. Coppola, T. Delmas, S. Epp, B. Erk et al., X-Ray Diffraction from Isolated and Strongly Aligned Gas-Phase Molecules with a Free-Electron Laser, Phys. Rev. Lett. 112, 083002 (2014).

[32] J. Yang, X. Zhu, T. J. A. Wolf, Z. Li, J. P. F. Nunes, R. Coffee, J. P. Cryan, M. Gühr, K. Hegazy, T. F. Heinz, K. Jobe, R. Li, X. Shen, T. Veccione, S. Weathersby, K. J. Wilkin, C. Yoneda, Q. Zheng, T. J. Martínez, M. Centurion et al., Imaging $\mathrm{CF}_{3} \mathrm{I}$ conical intersection and photodissociation dynamics with ultrafast electron diffraction, Science 361, 64 (2018).

[33] J. J. Larsen, I. Wendt-Larsen, and H. Stapelfeldt, Controlling the Branching Ratio of Photodissociation Using Aligned Molecules, Phys. Rev. Lett. 83, 1123 (1999).

[34] M. H. G. de Miranda, A. Chotia, B. Neyenhuis, D. Wang, G. Quéméner, S. Ospelkaus, J. L. Bohn, J. Ye, and D. S. Jin, Controlling the quantum stereodynamics of ultracold bimolecular reactions, Nat. Phys. 7, 502 (2011).

[35] K. P. Liu, Crossed-beam studies of neutral reactions: Statespecific differential cross sections, Annu. Rev. Phys. Chem. 52, 139 (2001).

[36] Y. Shagam, A. Klein, W. Skomorowski, R. Yun, V. Averbukh, C. P. Koch, and E. Narevicius, Molecular hydrogen interacts more strongly when rotationally excited at low temperatures leading to faster reactions, Nat. Chem. 7, 921 (2015).

[37] J. Karczmarek, J. Wright, P. Corkum, and M. Ivanov, Optical Centrifuge for Molecules, Phys. Rev. Lett. 82, 3420 (1999).

[38] D. M. Villeneuve, S. A. Aseyev, P. Dietrich, M. Spanner, M. Y. Ivanov, and P. B. Corkum, Forced Molecular Rotation in an Optical Centrifuge, Phys. Rev. Lett. 85, 542 (2000).

[39] L. Yuan, S. W. Teitelbaum, A. Robinson, and A. S. Mullin, Dynamics of molecules in extreme rotational states, Proc. Natl. Acad. Sci. USA 108, 6872 (2011).

[40] A. Korobenko, A. A. Milner, and V. Milner, Direct Observation, Study, and Control of Molecular Superrotors, Phys. Rev. Lett. 112, 113004 (2014).

[41] Y. Khodorkovsky, U. Steinitz, J.-M. Hartmann, and I. Sh. Averbukh, Collisional dynamics in a gas of molecular superrotors, Nat. Commun. 6, 7791 (2015).

[42] A. A. Milner, A. Korobenko, J. W. Hepburn, and V. Milner, Effects of Ultrafast Molecular Rotation on Collisional Decoherence, Phys. Rev. Lett. 113, 043005 (2014).

[43] A. A. Milner, A. Korobenko, K. Rezaiezadeh, and V. Milner, From Gyroscopic to Thermal Motion: A Crossover in the Dynamics of Molecular Superrotors, Phys. Rev. X 5, 031041 (2015).

[44] A. Owens, A. Yachmenev, and J. Küpper, Coherent control of the rotation axis of molecular superrotors, J. Phys. Chem. Lett. 9, 4206 (2018).

[45] I. Tutunnikov, E. Gershnabel, S. Gold, and I. S. Averbukh, Selective orientation of chiral molecules by laser fields with twisted polarization, J. Phys. Chem. Lett. 9, 1105 (2018).

[46] A. A. Milner, J. A. M. Fordyce, I. MacPhail-Bartley, W. Wasserman, V. Milner, I. Tutunnikov, and I. S. Averbukh, Controlled Enantioselective Orientation of Chiral Molecules with an Optical Centrifuge, Phys. Rev. Lett. 122, 223201 (2019).

[47] A. Yachmenev, J. Onvlee, E. Zak, A. Owens, and J. Küpper, Field-induced Diastereomers for Chiral Separation, Phys. Rev. Lett. 123, 243202 (2019).

[48] P. R. Bunker and P. Jensen, Chirality in rotational energy level clusters, J. Mol. Spectrosc. 228, 640 (2004).

[49] J. D. Pickering, B. Shepperson, L. Christiansen, and H. Stapelfeldt, Alignment of the $\mathrm{CS}_{2}$ dimer embedded in helium 
droplets induced by a circularly polarized laser pulse, Phys. Rev. A 99, 043403 (2019).

[50] I. MacPhail-Bartley, W. W. Wasserman, A. A. Milner, and V. Milner, Laser control of molecular rotation: Expanding the utility of an optical centrifuge, Rev. Sci. Instrum. 91, 045122 (2020).

[51] S. N. Yurchenko, W. Thiel, and P. Jensen, Theoretical ROVibrational energies (TROVE): A robust numerical approach to the calculation of rovibrational energies for polyatomic molecules, J. Mol. Spectrosc. 245, 126 (2007).

[52] A. Yachmenev and S. N. Yurchenko, Automatic differentiation method for numerical construction of the rotational-vibrational Hamiltonian as a power series in the curvilinear internal coordinates using the Eckart frame, J. Chem. Phys. 143, 014105 (2015).

[53] S. N. Yurchenko, A. Yachmenev, and R. I. Ovsyannikov, Symmetry adapted ro-vibrational basis functions for variational nuclear motion calculations: TROVE approach, J. Chem. Theory Comput. 13, 4368 (2017).

[54] A. A. A. Azzam, J. Tennyson, S. N. Yurchenko, and O. V. Naumenko, ExoMol molecular line lists - XVI. the rotationvibration spectrum of hot $\mathrm{H}_{2} \mathrm{~S}$, Mon. Not. R. Astron. Soc. 460, 4063 (2016).

[55] F. Weigend, F. Furche, and R. Ahlrichs, Gaussian basis sets of quadruple zeta valence quality for atoms $\mathrm{H}-\mathrm{Kr}$, J. Chem. Phys. 119, 12753 (2003).

[56] F. Weigend and R. Ahlrichs, Balanced basis sets of split valence, triple zeta valence and quadruple zeta valence quality for $\mathrm{H}$ to Rn: Design and assessment of accuracy, Phys. Chem. Chem. Phys. 7, 3297 (2005).

[57] A. Owens and A. Yachmenev, RichMol: A general variational approach for rovibrational molecular dynamics in external electric fields, J. Chem. Phys. 148, 124102 (2018).

[58] A. Yachmenev, L. V. Thesing, and J. Küpper, Laser-induced dynamics of molecules with strong nuclear quadrupole coupling, J. Chem. Phys. 151, 244118 (2019).

[59] I. N. Kozin and I. M. Pavlichenkov, Bifurcation in rotational spectra of nonlinear $\mathrm{AB}_{2}$ molecules, J. Chem. Phys. 104, 4105 (1996).

[60] N. V. Vitanov and B. M. Garraway, Landau-zener model: Effects of finite coupling duration, Phys. Rev. A 53, 4288 (1996).
[61] See Supplemental Material at http://link.aps.org/supplemental/ 10.1103/PhysRevResearch.3.023188 for simulated movies of joint $3 \mathrm{D}$ probabilities for the deuterium nuclei in $\mathrm{D}_{2} \mathrm{~S}$ created for rotational wave packets shown in Fig. 4. Attached are also respective animated velocity-map images (VMI) for the $\mathrm{D}^{+}$ion fragments.

[62] A. Korobenko, J. W. Hepburn, and V. Milner, Observation of nondispersing classical-like molecular rotation, Phys. Chem. Chem. Phys. 17, 951 (2015).

[63] T. Kierspel, J. Wiese, T. Mullins, J. Robinson, A. Aquila, A. Barty, R. Bean, R. Boll, S. Boutet, P. Bucksbaum, H. N. Chapman, L. Christensen, A. Fry, M. Hunter, J. E. Koglin, M. Liang, V. Mariani, A. Morgan, A. Natan, V. Petrovic et al., Strongly aligned molecules at free-electron lasers, J. Phys. B 48, 204002 (2015).

[64] D. Popova-Gorelova, J. Küpper, and R. Santra, Imaging electron dynamics with time- and angle-resolved photoelectron spectroscopy, Phys. Rev. A 94, 013412 (2016).

[65] O. Smirnova, Y. Mairesse, S. Patchkovskii, N. Dudovich, D. Villeneuve, P. Corkum, and M. Y. Ivanov, High harmonic interferometry of multi-electron dynamics in molecules, Nature (London) 460, 972 (2009).

[66] A. Trabattoni, J. Wiese, U. De Giovannini, J.-F. Olivieri, T. Mullins, J. Onvlee, S.-K. Son, B. Frusteri, A. Rubio, S. Trippel, and J. Küpper, Setting the photoelectron clock through molecular alignment, Nat. Commun. 11, 2546 (2020).

[67] M. Lapert, S. Guérin, and D. Sugny, Field-free quantum cogwheel by shaping of rotational wave packets, Phys. Rev. A 83, 013403 (2011).

[68] M. J. Murray, H. M. Ogden, and A. S. Mullin, Importance of rotational adiabaticity in collisions of $\mathrm{CO}_{2}$ super rotors with $\mathrm{Ar}$ and He, J. Chem. Phys. 148, 084310 (2018).

[69] H. Stapelfeldt, E. Constant, H. Sakai, and P. B. Corkum, Timeresolved Coulomb explosion imaging: A method to measure structure and dynamics of molecular nuclear wave packets, Phys. Rev. A 58, 426 (1998).

[70] P. B. Corkum, M. Y. Ivanov, and J. S. Wright, Subfemtosecond processes in strong laser fields, Annu. Rev. Phys. Chem. 48, 387 (1997). 